Acta Biol. Par., Curitiba, 35 (3-4): 185-195. 2006.

\title{
Atividade larvicida do extrato etanólico bruto da casca do caule de Magonia pubescens St. Hil. sobre Culex quinquefasciatus Say (Diptera, Culicidae)
}

\author{
Larvicidal activity of ethanol crude extract of
} the peel of the stem coat of Magonia pubescens St. Hil. against Culex quinquefasciatus Say (Diptera, Culicidae)

\section{Valeria de Oliveira Mendes Zanon ${ }^{1}$ \\ Heloísa Helena Garcia da Silva Regina Maria Geris dos Santos ${ }^{2}$ \\ IONIZETE GARCIA DA SILVA ${ }^{1,2}$}

Culex quinquefasciatus Say é a espécie mais importante na transmissão da Wuchereria bancrofti nas áreas em que esse nematóide apresenta periodicidade noturna. Apresenta distribuição trópicocosmopolita, sendo acentuadamente antropofílico, com comportamento altamente sinantrópico e endofílico, com hábitos hematofágicos noturnos, principalmente nas horas mais avançadas, coincidindo com o período de maior microfilaremia do helminto. Esse mosquito tornou-se o mais conhecido do ser humano, fundamentalmente por atacar e perturbar o seu repouso noturno (Consolin \& Oliveira , 1994; Foratini et al., 2000).

No Brasil, o $C x$. quinquefasciatus está muito bem adaptado ao meio urbano, desenvolvendo-se em criadouros naturais e artificiais, desde a água límpida até extremamente poluída, tornando-se uma

\footnotetext{
${ }^{1}$ Lab. Biologia, Fisiologia de Insetos e Xenodiagnóstico do Inst. Patologia Tropical e Saúde Pública (IPTSP), UFGO. ${ }^{2}$ Prof.Depto Microbiologia, Imunologia, Parasitologia e Patologia o IPTSP-UFGO. Endereço para correspondência: Rua Delenda Resende de Melo esq. Com a $1^{\text {a }}$ Avenida. Setor Universitário. - C.P. 131 - CEP 74605-050, Goiânia, GO. E-mail: ionizete@iptsp.ufg.br. Auxílio FUNAPE-2005, OPAS/PEA.
} 
praga urbana, apresentando maior densidade onde não há infraestrutura de saneamento básico. Nas áreas com focos de transmissão, vários métodos têm sido integrados para o controle do mosquito e da filariose bancroftiana, como métodos químicos, físicos e microbiológicos (Scaff \& Gueiros, 1968; Fraiha-Neto, 1993; CAlheiros et al., 1998; Fontes et al., 1994).

A filariose bancroftiana é também conhecida por elefantíase e constitui-se num sério problema de saúde na Ásia, África e nas Américas. Nessas áreas, estima-se que cerca de 120 milhões de pessoas estejam infectadas pela W. bancrofti. Esse Nematoda vive nos linfonodos e vasos linfáticos, causando obstrução e extravasamento de linfa, sendo responsável pelas manifestações clínicas mais graves de membros inferiores e da bolsa escrotal com edema linfático e queratinização cutânea (WHO, 1992 e 1994).

A bancroftose no Brasil apresenta uma distribuição urbana, localizada, principalmente nas cidades litorâneas. Na década de 50, essa parasitose distribuía-se em oito estados (Alagoas, Amazonas, Bahia, Maranhão, Pará, Pernambuco, Rio Grande do Sul, Santa Catarina). Após essa década, em função das medidas de controle utilizadas contra o vetor e tratamento dos microfilarêmicos, inquéritos mostram que a prevalência persiste nas regiões Norte e Nordeste, nas cidades de Belém, Maceió e Recife (Fontes et al.,2005; Dreyer \& CoelHo, 1997; Rocha et al., 2000). Os inquéritos sobre a bancroftose são localizados, por isso coloca em dúvidas as áreas consideradas atualmente indenes, nos estados do Amazonas, Bahia, Maranhão, Santa Catarina e Rio Grande do Sul (VIEIRA \& Coelno, 1998). Contudo estudos recentes, através do índice de infestação larvária do C. quinquefasciatus e pela microfilaremia sangüínea, evidenciaram situação mais problemática na cidade de Recife, com aumento da endemia (Dreyer \& Medeiros, 1990; Maciel et al., 1996 e 1999; Rocha et al., 2000, 1998; Vieira \& Coelho, 1998).

As ações de controle do C. quinquefasciatus são realizadas com inseticidas químicos, microbiológicos e manejo ambiental, apenas nos focos de transmissão. Com o aparecimento da resistência do mosquito a esses produtos (FAILloux et al., 1994; WIRTH et al., 1998), o controle da bancroftose tem sido centrado no diagnóstico, tratamento de microfilarêmicos e na conscientização sanitária da 
população (Scaff \& GueIros, 1968; Dreyer \& DReyer, 2000; VieIRA \& Coelho, 1998).

Inseticidas de origem botânica têm sido investigados como alternativa para controle do C. quinquefasciatus. Assim, CHAHAD \& Boof (1994) mostraram atividade larvicida de extratos de pimenta preta sobre o quarto estádio desse mosquito. A Azadirachta indica tem sido estudada por diversos autores, que demonstraram potencialidade dessa planta como larvicida para o $C$. quinquefasciatus (RAo et al., 1992; SAGAR \& SEHGAL,1996; SCOTT \& KaushiK, 1998; Su \& Mulla, 1998; El-Hag et al., 1999).

Com relação a plantas do Cerrado, o primeiro trabalho mostrando potencialidade larvicida do extrato bruto etanólico da Magonia pubescens foi o de SiLva et al. (1996) trabalhando com o Aedes aegypti e, GUIMARÃEs et al. (2001) realizaram bioensaios no campo com essa planta, correlacionando a atividade com o tipo de criadouro. Estudos mais detalhados da toxicidade foram feitos por SiLva et al. (2003) e ARRUdA et al. (2003a,b). Isso abriu perspectivas de estudos para o C. quinquefasciatus, na busca de novas alternativas para as ações de controle desse culicíneo.

\section{MATERIAL E MÉTODOS}

Coletaram-se as cascas do caule da $M$. pubescens, no município de Formosa, Goiás, que foram levadas ao laboratório de Bioatividade de Plantas do Instituto de Patologia Tropical e Saúde Pública (IPTSP), da Universidade Federal de Goiás (UFG), para a extração. As cascas foram colocadas em estufa com fluxo de ar forçado à $40^{\circ} \mathrm{C}$, para secagem, em seguida, trituradas em moinho de facas até atingir baixa granulometria e percoladas a frio. Esse processo consistiu em colocar cerca de 800 g do pó obtido da casca do caule, num béquer com capacidade para 2000 ml, no qual adicionou-se um litro de álcool etílico absoluto e misturou-se com agitador mecânico até completa homogeneização.

Cada béquer foi coberto com papel alumínio, para evitar a evaporação do álcool e uma possível interferência da luz, permanecendo em repouso por 72 horas. O sobrenadante foi filtrado em funil de vidro com papel filtro do tipo coador de café, descartável. Após cada filtragem o volume do béquer era completado com álcool 
para a seguinte percolação e esse procedimento foi repetido por quatro vezes.

O filtrado foi concentrado em evaporador rotativo e o extrato obtido colocado em placas de Petri para secagem, numa capela de exaustão, em temperatura ambiente. Após a evaporação do solvente, o extrato foi transferido para frasco de vidro âmbar e armazenado em dessecador, até sua utilização nos testes.

As larvas de $C$. quinquefasciatus utilizadas nos testes foram criadas de acordo com a metodologia de Silva et al. (1998), com água da rede pública de abastecimento, em câmara biológica climatizada a $28 \pm 1^{\circ} \mathrm{C}$, umidade de $80 \pm 5 \%$ e fotofase aproximada de 12 horas.

Os ensaios biológicos foram realizados em outra câmara climatizada similarmente à de criação. Foram feitas quatro réplicas de cada experimento, com seus respectivos controles, com o extrato bruto etanólico (e.b.e.) da casca do caule da M. pubescens, para determinar as concentrações letais: $\mathrm{CL}_{50}$ e $\mathrm{CL}_{99}$, para todos os estádios larvais de C. quinquefasciatus.

As soluções utilizadas para os testes, foram preparadas, pesandose o e.b.e. da casca do caule da M. pubescens, em balança analítica, com a precisão de 0,0001g. Em seguida, o e.b.e. foi dissolvido em água destilada, permanecendo em repouso cerca de uma hora, para facilitar a dissolução. Posteriormente, foi homogeneizado em agitador magnético por mais ou menos 10 minutos e o volume foi ajustado com água destilada. As soluções foram preparadas 24 horas antes da realização dos testes em copos plásticos, descartáveis, com $200 \mathrm{ml}$ de solução.

Foram utilizadas 20 larvas, recém eclodidas, de cada estádio para cada bioensaio e mesma quantidade para o controle. As larvas foram colocadas nas soluções com auxílio de pipetas plásticas descartáveis. O mesmo procedimento foi realizado para o controle, substituindo a solução por água destilada.

As leituras da mortalidade foram realizadas após 48 horas de exposição das larvas ao extrato, sendo consideradas mortas as larvas totalmente inertes, associado com o escurecimento do corpo e/ou da cápsula cefálica. As concentrações letais foram interpoladas pela Análise de Probit através do programa Statistical Product and Service Solution (SPSS). 


\section{RESULTADOS E DISCUSSÃO}

Das coletas feitas para iniciar a criação de $C$. quinquefasciatus observou-se uma grande variação, tanto nos tipos de criadouros quanto nas características da água, variando desde a água limpa até a extremamente poluída. Contudo, as larvas coletadas eram basicamente de criadouros com alto grau de poluição. Esse mosquito está tão adaptado a essas condições que, no laboratório, ao transferilas para bacias com água do sistema público, ocorria grande mortalidade. O desenvolvimento do C. quinquefasciatus em criadouros com água poluída sinaliza como um aspecto favorável às perspectivas de uso de extrato bruto de plantas no controle. Primeiro pela possibilidade de uso do produto na fase em que ele é mais barato, levando em consideração o custo-benefício e, segundo pela tecnologia mais simples de produção, possibilitando uso mais amplo sem impacto ambiental.

Os ensaios biológicos foram realizados com larvas de todos os estádios de $C$. quinquefasciatus, com o e.b.e. da casca do caule de M. pubescens que, após dissolvido apresentou coloração avermelhada e formação de espuma com forte tensão superficial. O e.b.e da $M$. pubescens demonstrou atividade larvicida para todos os estádios de $C$. quinquefasciatus. As $\mathrm{CL}_{50}$ encontradas para larvas de $1^{\circ}, 2^{\circ}, 3^{\circ}$ e $4^{\circ}$ estádios, foram, respectivamente, de 20, 30, 40 e $60 \mathrm{mg}$ para $100 \mathrm{ml}$ de água destilada. As $\mathrm{CL}_{99}$ para os mesmos estádios foram de 80, 80, 90 e 170 mg para 100ml de água destilada, respectivamente.

Trabalhos sobre a atividade larvicida de plantas para mosquitos são bastante heterogêneos, com concentrações letais muito variadas, como mostram as citações subseqüentes. Nas Filipinas, Monzon et al. (1994) estudaram o extrato bruto aquoso das plantas Lansium domesticum, A. indica, Eucaliptus globosus e Codiaeum variegatum. para $3^{\circ}$ e $4^{\circ}$ estádios do C. quinquefasciatus, apresentando concentrações letais extremamente elevadas, sendo a $\mathrm{CL}_{90}$ de 37, 28, 35 e 24 g/100 ml de água, respectivamente. Essa concentração é cerca de mil vezes superior a $\mathrm{CL}_{99}$ para $04^{\circ}$ estádio de C. quinquefasciatus encontrada neste trabalho.

Os resultados das concentrações letais são apresentadas na ordem crescente dos estádios larvais de C. quinquefasciatus como mostra a Figura 1. 


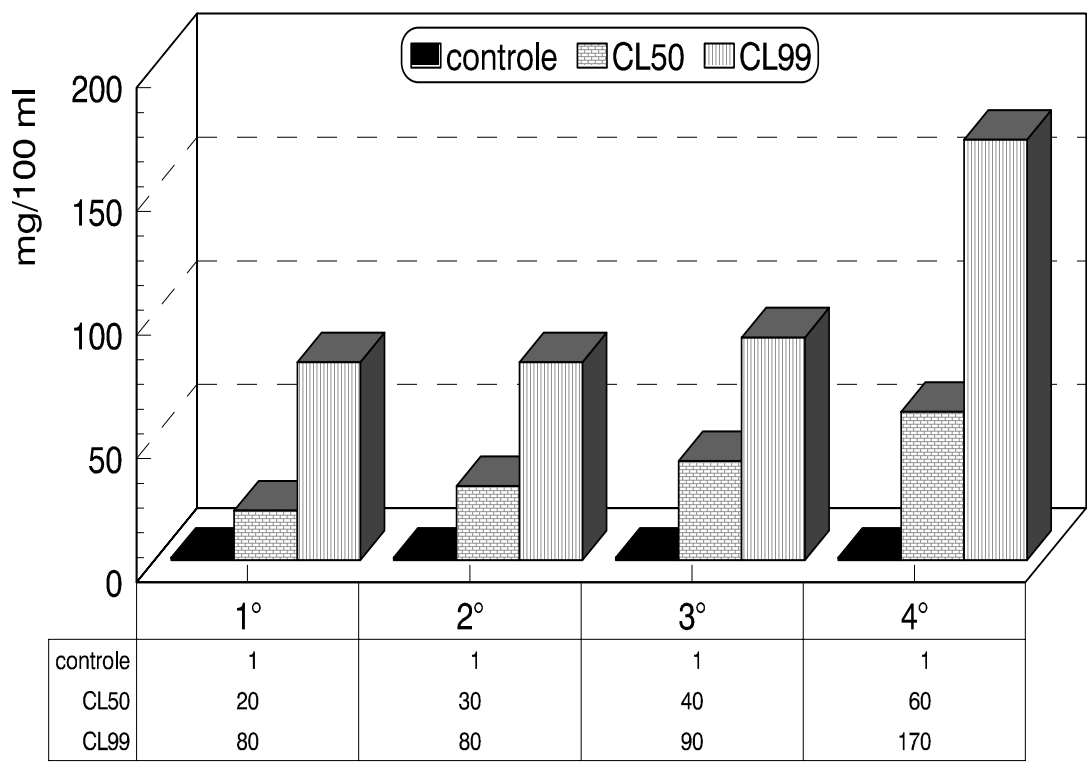

Estádios

Fig. 1. Concentrações letais $\mathrm{CL}_{50}$ e $\mathrm{CL}_{99}$ do extrato bruto etanólico da casca do caule de Magonia pubescens para todos os estádios larvais de Culex quinquefasciatus.

Concentrações letais inferiores às da $M$. pubescens obtidas com extrato bruto, para larvas do $4^{\circ}$ estádio do C. quinquefasciatus foram obtidas por СHAHAD \& BoOF (1994) com extratos de P. nigrum. Como esperado, essa planta também apresentou resultados inferiores aos dos princípios ativos isolados, nomilin e limonin, de uma planta da Índia, Citrus reticulata (JAYAPRAKASHA et al., 1997); do thymol e alfa-amyrin, da planta egípcia Thymus capitatus (MANsour et al., 2000), e na Tailândia, com a fração hexânica da Kaempferia galanga (Сноосноте et al., 1999).

$\mathrm{A} \mathrm{CL}_{50}$ do e.b.e. da casca do caule da $M$. pubescens para o terceiro estádio do C. quinquefascitus, foi $40 \mathrm{mg} / 100 \mathrm{ml}$, resultado similar aos obtidos por PizArro et al. (1999) com o extrato da Agave sisalana, que foi sugerida para uso no controle desse mosquito em função do tipo de criadouro e grau elevado de poluição da água onde as larvas se criam.

O e.b.e. da casca do caule da $M$. pubescens na concentração letal $\left(\mathrm{CL}_{99}\right)$ de $170 \mathrm{mg} / 100 \mathrm{ml}$ de água mostrou-se eficiente para 
todos os estádios larvais de C. quinquefasciatus. Resultados similares foram encontrados por SiLva et al. (1996) e GuIMARÃEs et al. (2001), que verificaram as concentrações letais do e.b.e. da casca do caule da $M$. pubescens de 140 e $150 \mathrm{mg} / 100 \mathrm{ml}$ de água destilada, para A. aegypti e A. albopictus. Esses resultados indicam a possibilidade de uso integrado dessa planta para combater esses mosquitos. A atividade inseticida do princípio ativo purificado e a análise estrutural da $M$. pubescens encontram-se em andamento, pelas técnicas de cromatografia e espectrometria de massa.

\section{RESUMO}

Foi estudada a atividade larvicida do extrato bruto etanólico (e.b.e) da casca do caule da Magonia pubescens St.Hil., sobre o Culex quinquefasciatus, na busca de novas alternativas para o controle do vetor mais importante da Wuchereria bancrofti. Após a coleta, as cascas foram dessecadas em estufa de ar forçado a $40^{\circ} \mathrm{C}$, moídas, percoladas a frio em etanol por 72 horas, filtradas, concentradas em evaporador rotativo e os extratos secados numa capela de exaustão à temperatura ambiente. $\mathrm{O}$ e.b.e obtido, foi dissolvido em água destilada para a realização dos testes, com todos os estádios larvais do C. quinquefasciatus. Os experimentos foram realizados em copos descartáveis com $200 \mathrm{ml}$ de solução, nos quais colocaram-se 20 larvas. Os mesmos procedimentos foram feitos para os controles, substituindo-se a solução por água destilada. Foram feitas 4 réplicas de cada experimento e seus respectivos controles. As observações da mortalidade foram realizadas após 48 horas de exposição das larvas à solução do extrato. Os experimentos foram realizados em câmara biológica climatizada a $28 \pm 1^{\circ} \mathrm{C}$, umidade relativa de $80 \pm 5 \%$ e fotofase de aproximadamente 12 horas. O e.b.e da $M$. pubescens demonstrou atividade larvicida para todos os estádios de $C$. quinquefasciatus. As $C_{50}$ encontradas para larvas de $1^{\circ}, 2^{\circ}, 3^{\circ}$ e $4^{\circ}$ estádios foram de $20,30,40$ e $60 \mathrm{mg} / 100 \mathrm{ml}$, respectivamente. Na mesma ordem as $\mathrm{CL}_{99}$ para os mesmos estádios foram de 80, 80, 90 e 170 mg/100ml de água destilada.

Palavras chave: Magonia pubescens, Culex quinquefasciatus,inseticida vegetal, filarióse bancroftiana .

\section{SUMARY}

Larvicidal activity of ethanol crude extract of the peel of the stem coat of Magonia pubescens St. Hil. against Culex quinquefasciatus Say (Diptera, Culicidae) - Bioassays were carried out in this work to verify larvicide activity of ethanol crude extract (e.c.e.) of the peel of the stem of Magonia pubescens St.Hil., on Culex quinquefasciatus, trying to find 
new alternatives to control the most important vector of the Wuchereria bancrofti. After being collected, the peels were desiccated in a forced air heather at $40^{\circ} \mathrm{C}$, ground, percolated in room temperature in ethanol for 72 hours, filtered, concentrated in rotating evaporator and desiccated in a chapel at room temperature. The e.c.e. obtained was solved in distilled water and tested for larvae from all instars of $C$. quinquefasciatus. The experiments were carried out in plastic glasses with $200 \mathrm{ml}$ of solution in each one, using 20 larvae to the test and the same number for the control. The mortality observations were made after 48 hours of exposition. The experiments were carried out in a weather controlled biological chamber at $28 \pm 2^{\circ} \mathrm{C}, 80 \pm 5 \%$ of relative humidity and 12 hours of photoperiod. The e.c.e. of the $M$. pubescens demonstrated larvicide activity against all instars of $C$. quinquefasciatus. The $\mathrm{LC}_{50}$ founded for $1^{\text {st }}, 2$, ${ }^{\text {nd }} 3^{\text {rd }}$ and $4^{\text {th }}$ larval instars was 20, 30, 40 and $60 \mathrm{mg} / 100 \mathrm{ml}$, respectively. The $\mathrm{LC}_{100}$ for the same instars was 80, 80, 90 and $170 \mathrm{mg} / 100 \mathrm{ml}$ of distilled water, respectively.

KEY WORDS: Magonia pubescens, Culex quinquefasciatus, botanical insecticide, bancroftian filariasis.

\section{RÉSUMÉ}

Les qualités larvecides de l'extrait brut de l'écorce (e.b.e.) de Magonia pubescens St. Hil, sur le Culex quinquefasciatus, dans le but de trouver des nouvelles alternatives pour le contrôle du plus important vecteur de la Wuchereria bancrofti. Après être ramassées, les écorces ont été déssechées dans des étuves d'air porté à $40^{\circ} \mathrm{C}$., broyées, macérées à froid dans de l'étanol pendant 72 heures, philtrées e concentrées dans un évaporateur rotatif. Les extraits obtenus ont été séchés dans un cloison du genre chapelle, à une température ambiante. On a fait la dissolution de l'e.b.e. obtenu, dans de l'eau distillée, pour la réalisation des tests à tous les stades larvaires du C. quinquefasciatus. Les expérimentations ont été faites dans des recipientes jettables, contenant $200 \mathrm{ml}$ de solution dans laquelle ont été mises 20 larves. Les mêmes procédés ont été pratiqués pour les contrôles, en substituant la solution par de l'eau distillée. Quatre (4) répétitions du procédé ont été faites, ainsi que ses respectifs contrôles. Les observations de la mortalité ont été réalisées après 48 heures d'exposition des larves à la solution de l'extrait. Les expérimentations ont été réalisées en chambres biologiques climatisées à $28^{\circ} \mathrm{C} \pm 1^{\circ} \mathrm{C}$, à une humidité relative de $80^{\circ} \mathrm{C} \pm 5^{\circ} \mathrm{C}$ et à une photophase d'environs 12 heures. L'e.b.e. de la Magonia pubescens a démontré l'activité larvecide à tous les stades du C. quinquefasciatus. Les CL50 trouvés pour les larves du 1er au 4ème stade, ont été de 20, 30, 40 et 60 mg/100ml, respectivement. Dans le même ordre,les CL99, pour les mêmes stades, ont été de 80, 80, 90 e 170 mg/100ml d'eau distillée.

Mots CLÉs: Magonia pubescens, Culex quinquefasciatus, insecticide végétal, filariose bancrotienne. 


\section{BIBLIOGRAFIA}

ARruda. W.; Oliveira G.M.C.; I.G. Silva. 2003a. Alterações morfológicas observadas em larvas de Aedes aegypti (Linnaeus, 1762) submetidas à ação do extrato bruto etanólico da casca do caule da Magonia pubescens. Entomol. Vect. 10: 47-60.

Arruda, W.; G.M.C. Oliveira; I.G. Silva. 2003b. Toxicidade do extrato etanólico de Magonia pubescens sobre larvas de Aedes aegypti. Rev. Soc. Bras. Med. Trop. 36:17-25.

Calheiros, C.M.L.; Fontes, G.; Willians, P.; Rocha, E.M.M., 1998. Experimental infection of Culex quinquefasciatus and Aedes (Stegomyia) aegypti with Wuchereria bancrofti. Mem. Inst. Oswaldo Cruz 93: 855-860.

Снанар, S. \& Boof, M.I.C., 1994. Efeitos de extratos de pimenta-preta sobre larvas de Culex (Culex) quinquefasciatus Say (Diptera: Culicidae). An. Soc. Entomol. Brasil 23: 13-18.

Сhoochote, W.; D. Kanjanapothi; A. Panthong; T. Taesotikul; A. Jitpakdi, U. Chaethong; B. Pitasawat. 1999. Larvicidal, adulticidal and repellent effects of Kaempferia galanga. Southeast Asian J. Trop. Med Public. Health 30: 470-476.

Consoli, R.A.G.B. \& R.L. Oliveira. 1994. Principais Mosquitos de Importância Sanitária no Brasil. Editora Fiocruz, Rio de Janeiro. $225 \mathrm{p}$.

Dreyer, G. \& G. Coelho. 1997. Filariose linfática: doença potencialmente eliminável. Cad. Saúde Pública 13: 537-543.

Dreyer, G. \& Z. Medeiros. 1990. Filariose linfática: ainda um desafio. Ciência Hoje 12: 6-7.

Dreyer, G. \& P. Dreyer. 2000. Bases para o tratamento da morbidade em áreas endêmicas de filariose bancroftiana. Rev. Soc. Bras. Trop. 33: 217-221.

EL-HAG, E.A.; A.H. NADI; A.A. Zaitoon. 1999. Toxic and growth retarding effects of three plant extracts on Culex pipiens larvae (Diptera: Culicidae).Phytother Res. 13: 388-392.

Failloux, A.B.; U.N.G. André; M. Raymond; N. PAsteur. 1994. Insecticide Susceptibility in Mosquitoes (Diptera: Culicidae) from French Polynesia. Entomol. Soc. America 31:639-644.

Fontes, G.; R.F. Braum; A. Fraia Neto; J.B.F. Vieira; S.S. Padilha; R.C. Rосна; E.M.M. Rосна. 2005. Filariose linfática em Belém, Estado do Pará, Norte do Brasil e aperspectiva de eliminação. Rev. Soc. Bras. Med. Trop.38:131-136. 
Fontes, G.; A.C. Brito; C.M.L. Calheiros; C.M.F. Antunes; E.M.M. Rocha. 1994. Situação atual da Filariose Bancroftiana na cidade de Maceió, Estado de Alagoas, Brasil. Cad. Saúde Públ. 10: 293-300.

Forattini, O.P.; I. KaKitani; R.C. Santos; K.M. Kobayashi; H.M. Ueno; Z. FernandÉz. 2000. Potencial sinantrópico de mosquitos Kertesia e Culex (Diptera: Culicidae) no Sudeste do Brasil. Rev.Saúde Pública 34: 565-569.

Fraina-Neto, H., 1993. Bancroftian filariasis in Belém, Pará. Possibilidades atuais de erradicação mediante a integração à campanha de novos métodos de controle da população de Culex quinquefasciatus. Cad. Saúde Pública 9: 458-465.

Guimarães, V.P.; I.G. Silva; H.H.G. Silva; C. Rocha. 2001. Atividade larvicida do extrato bruto etanólico da casca do caule de Magonia pubescens St. Hil. sobre o Aedes albopictus (Skuse, 1894) (Diptera, Culicidae). Rev. Patol. Trop. 30: 243-249.

Jayaprakasha, G.K.; R.P. Singh; J. Pereira; K.K. Sakariah. 1997. Limonoids from Citrus reticulata and their moult inhibiting activity in mosquito Culex quinquefasciatus larvae. Phytochemistry 44: 843846.

Maciel, A.; A.F. Furtado; K.B.F. Marzochi. 1999. Perspectiva da municipalização do controle da filariose linfática na região metropolitana do Recife. Cad. Saúde Pública, 15 (1): 195-203.

Maciel, A.R.A.; K.B. Marzochi; Z. Medeiros; A.B. Carvalho; L. Regis; W. SouzA; T. Lapa; A. Furtado. 1996. Epidemiological study of bancroftian filariasis in Recife. Mem. Inst. Oswaldo Cruz 91 (4): 449455.

Mansour, S.A.; Messeha, S.S.; El- Gengaini, S.E. 2000. Botanical biocides. 4. Mosquitocidal activity of certain Thymus capitatus constituents. J. Nat. Toxins 9: 49-62.

Monzon, R.B.; L.L.C. Lucson; A.S. Morales; F.E.S. Mutuc. 1994. Larvicidal potencial of five phiippine plants against Aedes aegypti (Linnaeus) and Culex quinquefasciatus (Say). Southteast Asian J. Trop. Med. Public Health 25: 755-759.

Pizzarro, A.P.; A.M. Oliveira-Filho; J.P. Parente; M.T. Melo; C.E. SANTOS; P.R. LiMA. 1999. Utilization of the waste of sisal industry in control of mosquito larvae. Rev Soc Bras. Med. Trop. 32: 23-9.

Rao, D.R.; R. Reuben; M.S. Venugopal; B.A. Nagasampagi; H. SCHMUtTERER. 1992. Evaluation of neem, Azadirachta indica, with and with and without water management, for the control of culicine mosquito larvae in rice-fields. Med. Vet. Entomol. 6: 318-324.

Rocha, E.M.M.; G. Fontes; A.C. Brito; T.R.C. Silva; Z. Medeiros; C.M.F. Antunes. 2000. Filariose bancroftiana em área urbanas do Estado de Alagoas , nordeste do Brasil; estudo em população geral. Rev. Soc. Bras. Med. Trop. 33 (6): 545-551. 
Sagar, S.K. \& S.S. Sehgal. 1996. Effects of aqueous extract of deoiled neem (Azadirachta Indica A. juss) seed Kernel and Karanja (Pongamia Glabra vent) seed Kernel against Culex quinquefasciatus. J. Commun Dis. 28: 260-269.

Su, T. \& M.S. Mulla. 1998. Ovicidal activity of neem products (azadirachtin) against Culex quinquefasciatus (Diptera Culicidae). J. Am. Mosq. Control Assoc. 14: 204-209.

SCAFF, L.M. \& Z.M. GueIros 1968. Erradicação da filariose. Endemias Rurais. Rev. Bras. Malariol. e Doenças Trop. 21: 604-613.

ScotT, I.M. \& N.K. KaushiK. 1998. The toxicity of Margosan-O, a product of neem seeds, to selected target and nontarget aquatic invertebrates. Arch. Environ. Contam. Toxicol. 35: 426-431.

SiLva, H.H.G.; I.G. SiLva; K.S. LiRA. 1998. Metodologia de criação, manutenção de adultos e estocagem de ovos de Aedes aegypti (Linnaeus, 1762) em laboratório. Rev Pat Trop 27: 51-63.

Silva, I.G.; A.H. Santos; P.H. Ferri; F.B.N. Alves; R.Q. Melo; L. Peixoto; H.H.G. Silva; C.N. Elias; E. IsaC; K.S. LiRA; M.F. Camargo. 1996. Ação larvicida de extrato bruto etanólico de Magonia pubescens St. Hil (tingui-do-Cerrado), sobre o Aedes aegypti (Lin.) em laboratório. Rev. Pat. Trop. 25: 51-59.

Silva, I.G.; V.P. Guimarães, C.G. Lima; H.H.G. Silva; C.N. Elias; C.M. Mady; V.V.M. Silva; A.P. Nery; K.R. Rocha; C. Rocha; E. Isac. 2003. Efeito larvicida e toxicológico do extrato bruto etanólico de Magonia pubescens St. Hil, sobre o Aedes aegypti (Diptera, Culicidae) em criadouros artificiais. Rev. Pat. Trop. 32: 73-86.

VieiRA, J.B.F.\& G.E. Coelho. 1998. Filariose; aspectos epidemiológicos e de controle. Rev. Soc. Bras. Med. Trop. 31 (2): 79-83.

Wirth, M.C.; A. Delécluse; B.A. Federici; W.E. Walton. 1998. Variable cross-resistence to Cry 11B from Bacillus thuringiensis subsp. jegathesan in Culex quinquefasciatus (Diptera; Culicidae) Resistant to single or multiple toxins of Bacillus thuringiensis subsp. israelensis. Appl. Envir. Microbiol. 64: 4174-4179.

World Health Organization. 1992. Limphatic filariasis: the disease and its control. Fifth report of the expert committee on filariasis. WHO Techinical Report Series 821: 1-71.

World HEALth ORGanizatIon. 1994. Strategies for control of lymphatic filariasis infection and disease: Report of a CTC/TDR/FIL/PENANG/94.1.

Recebido em 5 de dezembro de 2005. 